REVIEW

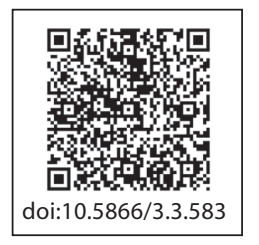

\title{
A New Paradigm in Autoimmunity-Role In Periodontal Disease
}

\author{
Snophia Suresh ${ }^{1}$
}

Reader $^{1}$

Dept. of Periodontics, Thaimoogambigai Dental College, Golden George Nagar, Chennai-107

\section{Article Info}

Received: April 13, 2011

Review Completed: May, 15, 2011

Accepted: June, 16, 2011

Available Online: October, 2011

(c) NAD, 2011 - All rights reserved

\begin{abstract}
:
Chronic diseases, such as periodontal disease and rheumatoid arthritis, are characterized by a robust immune response resulting in unresolved inflammation. Inflammation is mediated by proinflammatory cytokines; recently, a novel subset of T-helper (Th) cells was identified that plays a crucial role in inflammation and autoimmune disease. This population secretes several proinflammatory cytokines, including the novel cytokine interleukin (IL)-17, and, hence, has been termed "Th17." Inflammatory cytokines are implicated in the progression of localized chronic infections, such as periodontal disease, and in serious systemic pathologies, such as diabetes, chronic obstructive pulmonary disease, and cardiovascular disease. Therapeutics that antagonize inflammatory cytokines ameliorate inflammation and bone loss and may have broader implications for individuals with systemic diseases in which inflammation and autoimmunity predominate.
\end{abstract}

Key words: Interleukin-17, T-helper cells

\section{Introduction}

Periodontal disease is risk factor for a host of systemic pathologies ranging from exacerbated cardiovascular disease to diabetes. It is clear that inflammatory cytokines constitute a key link between periodontal disease and systemic disease. Thus understanding the multilayered mechanism of inflammation in periodontal disease has significant therapeutic implications. Inflammatory cytokines such as tumor necrosis factor-alpha and Interleukin1 beta(IL-1) and -6 are produced by many cell types. Exciting new evidence has emerged relating to the production of inflammatory cytokines by a novel subset of CD4+ T-helper (Th) cells. ${ }^{1}$ This population plays an essential role in regulating autoimmune disease and inflammation by secreting a novel proinflammatory cytokine, Interleukin-17(IL-17); hence these cells are termed "Th17".

Email for correspondence: suresh_sno@yahoo.com

\section{IL-17 -A Novel Cytokine Family}

There are more than 200 cytokines in mammals, which exert a vast range of biologic activities on numerous biologic systems. Cytokines are subdivided into families based primarily on receptor structure and IL-17 is the new grouping to be described. ${ }^{2}$ Members of the IL-17 and -17R families are unique in amino acid sequence, with little homology to other cytokine classes. Six IL-17 family members and five receptors have been identified which are conserved in rodents and humans. (Table)

\section{T-helper-17 Cell}

It has long been established that CD4+ Th cells are divided into functional subsets that regulate the separate types of immune responses. Whereas Th subset1(Th1) cells secrete interferon-gamma (IFNgamma) in response to differentiation cues from IL12 and mediate cellular immunity, Th subset2 (Th2) 
cells secrete IL-4 upon differentiation in the presence of IL-4 and activate allergic and humoral responses.

For several decades, the Th1/Th2 paradigm held its way and was used to describe the progression of inflammatory and autoimmune disorders. Although IL-17 was known for a long time, it was thought to be produced mainly by the Th1/Th0 cells. Th- 17 cells were identified as a separate phenotype only recently. ${ }^{3}$ Recently a novel subset of Th cells, distinct from the Th1 and Th2 subsets, was discovered that explains some of the shortcomings of the Th1/Th2 model. Initially this Th population was identified based on the insight that IL-12 family cytokine, IL-23 could drive T-cells to secrete IL-17, rather than IL-4 or IFN-gamma. More recent studies showed that Th17 cells arose in the presence of transforming growth factor - beta., IL-6, and - 1 beta and were expanded specifically by IL-23. These cells secrete IL-17 in addition to IL-17F, -22 , and -6 and TNF-alpha, hence are designated "Th17". (Fig-1)The signature cytokine of Th17 cell is IL-17A, but they are known to produce IL-21 and IL-22. IL-17A is thought to be important for the recruitment of neutrophils. It is thus believed that Th17 cells have evolved primarily to provide inflammatory protection against the extracellular pathogens. They are also believed to be involved in inflammatory bone disorders such as rheumatoid arthritis.

\section{Inducing Signal for T-helper-17 Cell}

Th17 cells are thought to develop in the presence of transforming growth factor-beta in synergy with IL-6 and/or IL-1. In addition, IL-21 and IL-23 signals are necessary for expansion and stabilization of their phenotype. ${ }^{4}$ Of these inductive signal, IL-23 (a member of IL-12 family) has been identified in the inflamed tissues as part of the early chemokine/cytokine infiltrate. The presence of IL-6, IL-1 and TGF-beta in the chronically inflamed gingival tissue is well documented and would seem to create an environment conducive to Th17 development. It is agreed that the master regulator of Th17 development is retinoic acid-related orphan receptor.

\section{T-helper-17 Cells in Periodontal Disease:}

Th17 cells have been identified in the periodontal tissues. There is however, a considerable difference between the murine and human cell types. Th17 has been proposed to exert both pro inflammatory and anti-inflammatory functions.

It was demonstrated that Porphyromonas gingivalis outer membrane protein induced a significant increase in the production of IL-17. ${ }^{\mathrm{IL}-17}$ has been shown to stimulate epithelial, endothelial and fibroblastic cells to produce IL-6, IL-8 and PGE2. ${ }^{6}$ In addition, IL-17 induces receptor activator of nuclear factor-kappa B ligand (RANKL) production by osteoblasts and thus influence osteoclastic bone resorption. Although there are conflicting reports, several authors have identified IL-17 in the gingival crevicular fluid. ${ }^{7}$ Moreover peripheral blood mononuclear cells from gingivitis and periodontitis patients stimulated with a Porphyromonas gingivalis antigen secreted IL-17. The level of IL-17 was higher in the gingivitis patients whereas the number of IL17 -positive samples was higher in the periodontitis patients. ${ }^{5}$ Thus numerous studies indicated a potential role for IL-17 mediated inflammation in the initiation and progression of the periodontal lesion suggesting that Th17 cells may contribute to the pathogenic tissue destruction that occurs in periodontal disease.

IL-17 seems to blur the lines between innate and adaptive immunity because it is secreted by the adaptive immune system and mediates activities typical of innate inflammatory effectors, such as TNFalpha and IL-1 beta. Specifically IL-17 differs from classic inflammatory cytokines in that it is produced largely by activated memory T-cells rather than innate immune cells. Despite its production by Tlymphocytes, IL-17 plays a crucial role in innate immunity because its secretion triggers production of numerous chemokines resulting in neutrophil and macrophage recruitment. ${ }^{8}$ In this way, IL-17 mediates cross-talk between the adaptive and innate immune systems, allowing for orchestration of an effective immune response.

A major advance in understanding the molecular link between the immune system and bone came with the discovery of receptor activator of nuclear factor kappa B (RANK) and RANK ligand(RANKL)., TNF superfamily receptors that are central to the regulation of osteoclastogenesis. RANKL is induced 
by inflammatory cytokines, such as IL-17, -1 beta, and - 6 and TNF-alpha, on osteoblastic stromal cells, osteoblasts and bone marrow stromal cells. Binding of RANKL to its receptor RANK drives differentiation to a mature osteoclast phenotype with bone resorbing capacity. Osteoprotegrin (OPG) is a soluble decoy receptor that binds $R A N K L$, inhibiting its interaction with RANK. Accordingly, OPG functions as a bone-protective cytokine preventing osteoclast maturation and subsequent bone resorption. Several studies demonstrated that IL-17, like many inflammatory cytokines, drives bone remodeling, thus favouring bone loss. First IL-17 can modulate the RANKL/OPG ratio; IL-17 increases RANKL expression and concomitantly decreases OPG expression in osteoblastic cells in vitro and in vivo, resulting in enhanced formation of osteoclasts and bone erosion in a mouse model of arthritis. Moreover, in conjunction with TNF-alpha, IL-17 was shown to increase bone resorption. Th17 cells also expressed higher levels of RANKL than TH1 cells. ${ }^{9}$

Evidence also suggests that chronic inflammation of the periodontium has far-reaching consequences for overall health and inflammatory cytokines, such as IL-17, may be important links between oral and systemic disease.

\section{Interleukin-17 in Autoimmunity}

Numerous studies demonstrated the importance of IL-17 in autoimmunity. In this regard, in collagen-induced arthritis, an IL-17 neutralizing antibody results in reduced joint inflammation, cartilage destruction, and bone erosion. Analysis of synovial and serum samples for IL-17 showed elevated levels of IL-17 in inflammatory diseases, such as periodontal disease, as well as in autoimmune conditions such as Rheumatoid arthritis, psoriasis, inflammatory bowel disease, and multiple sclerosis. ${ }^{10}$

\section{Anti Cytokine Therapies}

It is striking that most successful anticytokine therapy has targeted the Th17 inflammatory axis. ${ }^{11}$ For example, IL-17 drives expression of IL-1 and TNFalpha, and agents that target the type II IL-1beta receptor and soluble TNF-alpha type I are highly effective in RA therapy. However no single therapy is effective for all patients suggesting that blocking other cytokines or their receptors may be a valuable therapeutic alternative in these individuals. IL-17 receptor (IL-17R) has three subunits IL-17RA, IL-17RF and IL-17RC. The IL-17R is an obvious candidate in this regard because of its role in pathogenic bone loss. Therapeutic agents that antagonize signaling through the IL-17R complex may serve as useful clinical option for individuals who are unresponsive to available drug regimens.

In recent years, a paradigm shift has occurred regarding how cytokine receptors oligomerize and deliver signals. Previously ligation of cytokine to its corresponding receptor was believed to be the initiating event in receptor oligomerization and subsequent signal transduction. Recent evidence indicated that several cytokine receptors form complexes even in the absence of ligand. These findings have led to the discovery of special motifs within cytokine receptors that direct specific receptor preassembly, therby preventing formation of nonproductive receptor complexes. Such motifs are the"pre-ligand binding assembly domain"(PLAD) located within the extracellular domain. ${ }^{12}$ Thus identification of the IL-17R PLAD and subsequent design of agents to disrupt IL-17R complexes could reduce localized and/or systemic inflammation, leading to additional treatment options for inflammatory diseases.

\section{Conclusion}

In this regard, the $\mathrm{IL}-17$ signaling complex is a compelling therapeutic target because of its prominent role in bone loss and autoimmunity. Through careful study of the interactions between IL-17 and its receptor complex, mechanisms of inflammatory signaling can be identified and ultimately targeted with rational therapeutics. However, therapeutics targeting chronic inflammation, in conditions such as RAand MS, may have unpredictable and even detrimental effects on the health of the periodontium.

\section{References}

1. Dong C. Diversification of T-helper-cell lineages: Finding the family root of IL-17-producing cells. NatRev Immunol 2006;6:329-333.

2. Aggarwal S, Gurney AL. IL-17: A prototype member of an emerging family. J Leukoc Biol 2002;71:1-8. 
3. Cua DJ, Sherlock J, Chen Y, Murphy CA, Joyce B, Seymour B et.al. Interleukin-23 rather than interleukin-12 is the critical cytokine for autoimmune inflammation of the brain. Nature2003;421:744-748.

4. Weaver CT, Hatton RD, Mangan PR, Harrington LE. IL-17 family cytokines and the expanding diversity of effector Tcell lineages. Annu Rev Immunol 2007;25:821-852.

5. Oda T, Yoshie H, Yamazaki K. Porphyromonas gingivalis antigen preferentially stimulates $\mathrm{T}$ cells to express IL-17 but not receptor activator of NF-kappaB ligand in vitro. Oral Microbiol Immunol 2003:18:30-36.

6. Fossiez F, Djossou O, Chomarat $\mathrm{P}$, et al. T cell interleukin-17 induces stromal cells to produce proinflammatory and hematopoietic cytokines. J Exp Med 1996;183:2593-2603.

7. Takahashi K, Azuma T, Motohira H, Kinane DF, Kitetsu S. The potential role of interleukin-17 in the immunopathology of periodontal disease.J Clin Periodontol 2005;32:369-374.

8. Yao Z, Painter SL, Fanslow WC, et al. Cutting edge: Human IL-17: A novel cytokine derived from T cells. J Immunol 1995; 155:5483-5486. -386.

9. Sato K, Suematsu A, Okamoto K, et al. Th17 functions as an osteoclastogenic helper $\mathrm{T}$ cell subset that links $\mathrm{T}$ cell activation and bone destruction.J Exp Med 2006;203:26732682.
10. Nakae S, Saijo S, Horai R, Sudo K, Mori S, Iwakura Y. IL-17 production from activated $T$ cells is required for the spontaneous development of destructive arthritis in mice deficient in IL-1 receptor antagonist. Proc Natl Acad Sci USA 2003; 100:5986-5990.

11. Kikly K, Liu L, Na S, Sedgwick JD. The IL-23/Th(17)axis: Therapeutic targets for autoimmune inflammation. Curr Opin Immunol 2006;18:670-675.

12. Deng GM, Zheng L, Chan FK, Lenardo M. Amelioration of inflammatory arthritis by targeting the preligand assembly domain of tumor necrosis factor receptors. Nat Med 2005;11:1066-1072.

Table-1 The IL-Cytokine Family

\begin{tabular}{cl} 
LIGAND & RECEPTOR \\
\hline IL-17A & IL-17RA \\
\hline IL-17B & IL-17RB \\
\hline IL-17C & IL-17RC \\
\hline IL-17D & IL-17RD \\
\hline IL-17E & IL-17RE \\
\hline IL-17F & \\
\hline VIL-17 &
\end{tabular}

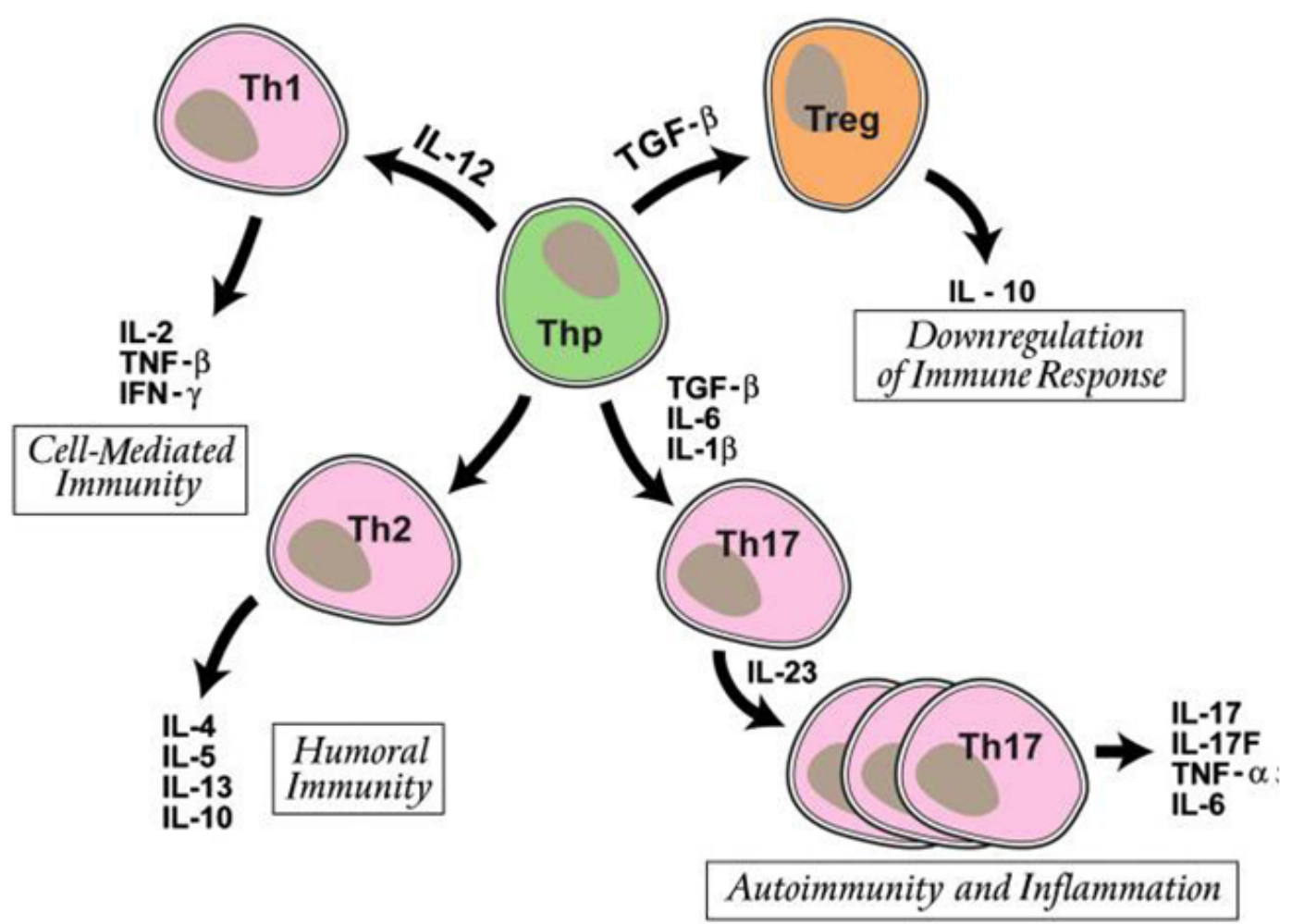

Figure-1. Interleutin - 17 - A New Paradigm in Autoimmunity - Role in Periodontal Disease. 